\title{
Knowledge Network Driven Coordination and Robust Optimization to Support Concurrent and Collaborative Parameter Design
}

\author{
Jie $\mathrm{Hu},{ }^{1, *}$ Yinghong Peng ${ }^{1}$ and Guangleng Xiong ${ }^{2}$ \\ ${ }^{1}$ Institute of Knowledge Based Engineering, School of Mechanical Engineering \\ Shanghai Jiao Tong University, Shanghai, 200240, PR China \\ ${ }^{2}$ State CIMS Engineering Research Center, Department of Automation \\ Tsinghua University, Beijing,100080, PR China
}

\begin{abstract}
This study presents a parameter coordination and robust optimization approach based on knowledge network modeling The method allows multidisciplinary designer to synthetically coordinate and optimize parameter considering multidisciplinary knowledge. First, a knowledge network model is established, including design knowledge from assembly, manufacture, performance, and simulation. Second, the parameter coordination method is presented to solve the knowledge network model, monitor the potential conflicts due to engineering changes, and obtain the consistency solution space corresponding to the given knowledge. Finally, the robust parameter optimization model is established, and genetic arithmetic is used to obtain the robust optimization parameter. A design instance is introduced to show the validity of this method.
\end{abstract}

Key Words: knowledge network, parameter coordination, robust design, multidisciplinary, concurrent and collaborative design.

\section{Introduction}

The overall performance of a complex product generally depends on a number of specifications distributed in multi-teams from different disciplines, such as mechanics, cybernetics, dynamics, and so forth. Many researchers paid attention to multidisciplinary collaborative design optimization approaches. Fonseca and Fleming [1] and Coello [2] provided comprehensive reviews of GA-based multiobjective optimization approaches. Fonseca and Fleming [3] developed the multi-objective genetic algorithm (MOGA) approach. Narayanan and Azarm [4] proposed MOGA-NA that is an extension of the original MOGA by Fonseca and Fleming [3] and made some improvements over the original MOGA. Both MOGA and MOGA-NA are able to produce a set of pareto solutions in one GA run without converting the multiple objectives to a single objective and, therefore, they show the great potential in obtaining the Pareto solutions in an efficient way. Narayana and Azarm [4] and Kurapati et al. [5] presented a penalty method to handle constraint in a MOGA. Besides the penalty methods, there exist many

*Author to whom correspondence should be addressed. E-mail: hujie@sjtu.edu.cn

Figure 4 appears in color online: http://cer.sagepub.com other approaches to handle constraints. Some of the approaches are the fuzzy logic approach by Le [6], and the immune system approach by Hajela and Lee [7]. A comprehensive survey of these constraint-handling techniques can be found in Coello [8] and Michalewicz [9]. Bloebaum et al. [10] and Renaud and Tappeta [11] presented the concurrent subspace optimization approach. Kroo et al. [12] and Braun et al. [13] presented the collaborative optimization approach. Kroo [14] provided a complete review of the multidisciplinary design optimization architectures.

However, the essence of a multidisciplinary collaborative design process is how to coordinate all the constraints distributed in various disciplines to maintain the consistency between product specifications and design variables. Serrano and Gossard [15] developed a graphical theory approach to evaluate the approximation degree of a set of constraints. Kannapan and Marshek [16] introduced a design diagram to represent parametric design problems and game theory to solve conflicts when constraints were violated. Kusiak and Wang [17] proposed a reduction algorithm for qualitative constraint networks that can describe the effect of perturbances from one variable on the other variables. Chen and Lewis [18] used robust design theory to deal with the coupling relationships among subsystems, and a model based on game theory took effect when the subsystem constraints conflicted. Lottaz and Robert [19] 
put forward a concept of solution space consistency, which replaced a single solution by solution space in the design process. Chang et al. [20] used Taguchi's parameter design concept to support teams communicating about sets of possibilities and to make decisions that are robust against variations in the designs carried out by other team members.

The first problem with these researchers is that they focused on the coordination and optimization in multidisciplinary collaborative design. However, the most important problem for multidisciplinary collaborative design is how to obtain the design knowledge from a multidisciplinary domain. The second problem with these researchers is that few papers have addressed the coordination and optimization simultaneously.

A knowledge network modeling, coordination, and robust optimization method inherited from concurrent and collaborative design theories are introduced in this study. It collects the design knowledge to construct a formulated model based on knowledge networks; uses interval boxes to describe the parameters' uncertainties to enhance the design robustness; and a parameter coordination algorithm framework is put forward to refine the design parameter intervals. With the help of this method, designers can detect the potential conflicts due to engineering changes, obtain the consistent solution space corresponding to the given specifications, and determine design variables within the solution space using multiobjective design optimization finally.

\section{Knowledge Network Modeling}

Figure 1 shows the 'time dimension' concurrence and 'space dimension' collaboration in the development process of a complex product. From the point of view of concurrent engineering, the process of manufacture and assembly directly affects product cost and performance. From the point of view of collaborative design, product design substantively is a multidisciplinary design including mechanics, cybernetics, dynamics, and so on.

\subsection{Knowledge Network Modeling for Concurrent Design}

\subsubsection{DESIGN KNOWLEDGE FROM ASSEMBLY}

Design knowledge from assembly includes parameter constraints, variation functions, and tolerance constraints.

- Parameter constraints

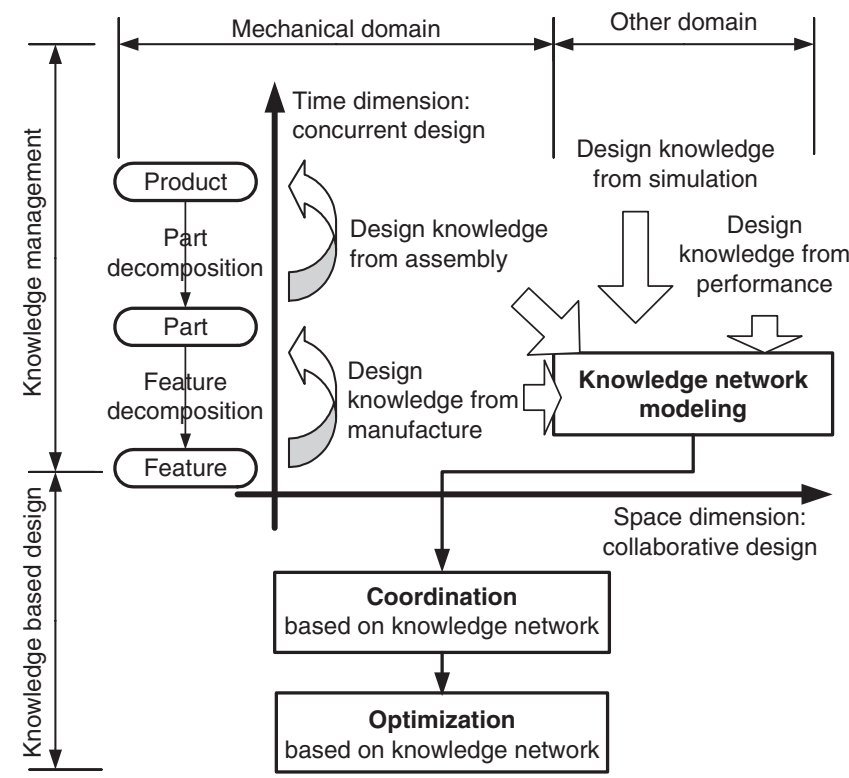

Figure 1. The knowledge network model for coordination and optimization to support concurrent and collaborative design.

Definition 1. Loop circuit - LC. Loop circuit is a looped subgraph with features as nodes and geometric constraints as arcs. That is

$$
\mathrm{LC}=G(\boldsymbol{V} ; \boldsymbol{E})
$$

such that StartNode $($ LC) $=$ EndNode $($ LC)

where $\boldsymbol{V}$ is the set of features and $\boldsymbol{E}$ is the set of geometric constraints.

In a loop circuit, the relation between nominal features is expressed as parameter constraints. For example, as in the assembly in Figure 2(a). There are two loop circuits in Figure 2(b), the parameter constraints are

$$
\left\{\begin{array}{l}
g_{1}=Y_{1}-\left(X_{2}-X_{4}\right)=0 \\
g_{2}=Y_{2}-\left(-X_{1}-X_{2}-X_{3}+X_{5}\right)=0 .
\end{array}\right.
$$

- Variation functions

Definition 2. Screw parameter. In 3D space, geometric variation includes six degrees of freedom (DOF): three independent translations $\left(T_{x}, T_{y}, T_{z}\right)$ and three independent rotations $\left(R_{x}, R_{y}, R_{z}\right)$. In the system of coordinates $\{O\}$, geometric variation is mathematically expressed by the six screw parameters: differential translations $u_{i}, v_{i}$, $w_{i}$ along $x, y, z$ axes, and differential rotation angles $\alpha_{i}$, $\beta_{i}, \gamma_{i}$ about $x, y, z$ axes.

Definition 3. Variation functions. The variation functions aim to establish the relation between all the variations in a loop circuit and assembly function requirement (AFR), and can be expressed as:

$$
\begin{aligned}
f_{k}= & c_{\alpha k} \cdot \alpha_{i}+c_{\beta k} \cdot \beta_{i}+c_{\gamma k} \cdot \gamma_{i}+c_{u k} \cdot u_{i} \\
& +c_{v k} \cdot v_{i}+c_{w k} \cdot w_{i}
\end{aligned}
$$




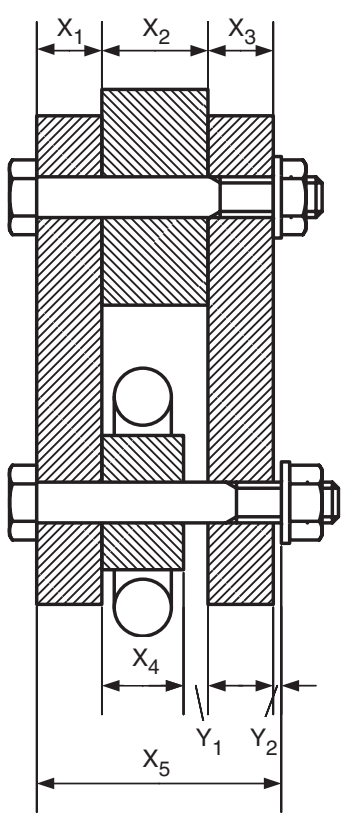

(a) The assembly

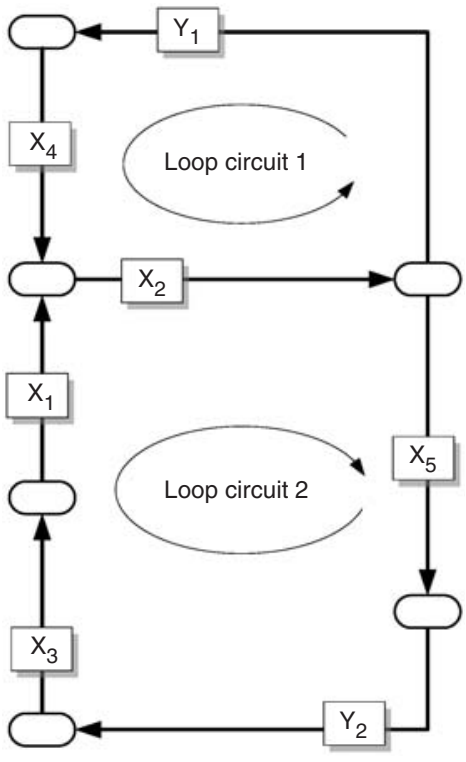

(b) The loop circuit
Figure 2. An illustration of loop circuit.

where $\alpha_{i}, \beta_{i}, \gamma_{i}, u_{i}, v_{i}, w_{i}(i=1, \ldots, n)$ are screw parameters of variation in loop circuit, $\alpha_{0}, \beta_{0}, \gamma_{0}, u_{0}, v_{0}, w_{0}$ are screw parameters of assembly function requirement, and $c_{\alpha k}$, $c_{\beta k}, c_{\gamma k}, c_{u k}, c_{v k}, c_{w k}$ are coefficients.

The variation functions in a loop circuit include the rotational functions and the translational functions, and can be expressed as:

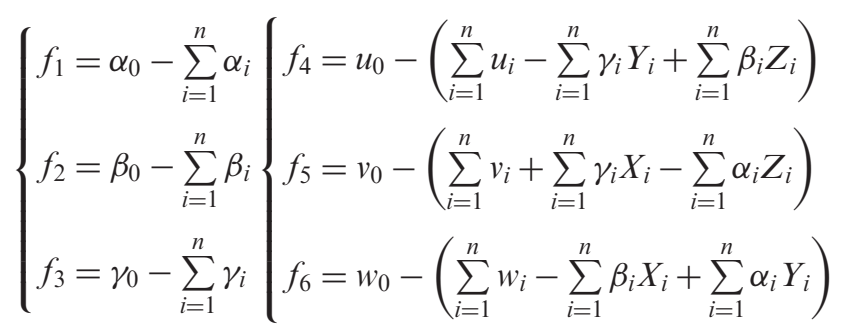

where $u_{i}, v_{i}, w_{i}, \alpha_{i}, \beta_{i}, \gamma_{i}$ are screw parameters of the $i$ th variation in loop circuit (LC), and $u_{0}, v_{0}, w_{0}, \alpha_{0}, \beta_{0}, \gamma_{0}$ are screw parameters of variation corresponding to assembly function requirement in loop circuit (LC).

\section{- Tolerance constraints}

Definition 4. Tolerance constraints. The tolerance constraints aim to establish the relation between the tolerances zone (given tolerance $t_{i}$ ) and the specified screw parameters $\left(\alpha_{i}, \beta_{i}, \gamma_{i}, u_{i}, v_{i}, w_{i}\right)$ of corresponding variation, and can be expressed as:

$$
h_{i}\left(\alpha_{i}, \beta_{i}, \gamma_{i}, u_{i}, v_{i}, w_{i} ; t_{i}\right) \leq 0, \quad i=0,1, \ldots, n .
$$

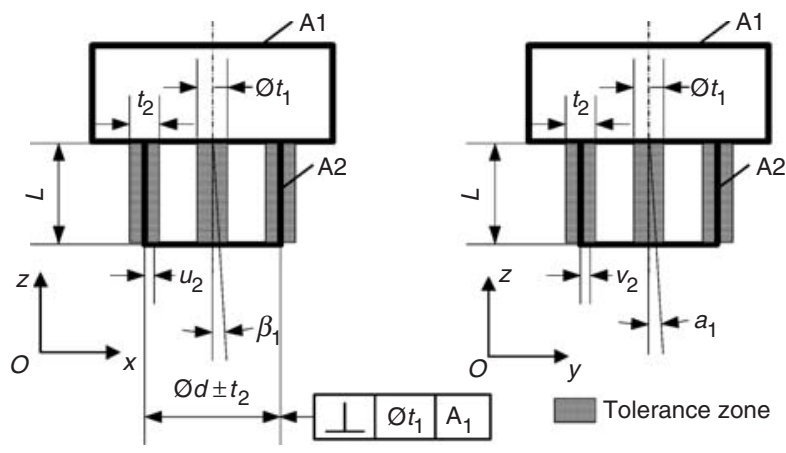

Figure 3. An illustration of tolerance constraints.

Figure 3 shows an example to illustrate how to specify tolerance constraints. For example perpendicularity, the tolerance zone is cylindrical, and the tolerance value is $t_{1}$. The corresponding screw parameter of variation is $\left(\alpha_{1}, \beta_{1}\right)$, and then tolerance constraint can be represented as:

$$
h\left(\alpha_{1}, \beta_{1} ; t_{1}\right)=\left[\left(L \cdot \alpha_{1}\right)^{2}+\left(L \cdot \beta_{1}\right)^{2}\right]-\left(\frac{t_{1}}{2}\right)^{2} \leq 0 .
$$

\subsubsection{DESIGN KNOWLEDGE FROM MANUFACTURE}

In design phase, a variation factor in a manufacture process should be considered, which is expressed as the manufacturing cost - parameter and tolerance function, which describes the relationship between the parameter and tolerance values and manufacturing cost.

Definition 5. Relative manufacturing cost. The manufacturing cost of casting processes with very loose tolerance $(>0.5 \mathrm{~mm})$ is used as the reference and assigned a value of 1 . The manufacturing cost increase value $(\%)$ is often called relative manufacturing cost.

Based on the empirical cost - parameter and tolerance data frequently used in manufacturing processes, the relative manufacturing cost - parameter and tolerance functions are established, such as

- Manufacturing cost function for cylindrical (shaft) feature and cylindricity tolerance:

$$
\begin{aligned}
f_{1}\left(t_{i}, d_{i}, l_{i}\right)= & 84.708-277.022 \cdot t_{i}+0.0938 \cdot d_{i}-0.00396 \cdot l_{i} \\
& +163.566 \cdot t_{i}^{2}+0.00135 \cdot d_{i}^{2}+0.000507 \cdot l_{i}^{2} \\
& +0.0262 \cdot t_{i} \cdot d+0.0208 \cdot t_{i} \cdot l_{i}+0.000245 \cdot d_{i} \cdot l_{i} \\
& +110.645 \cdot t_{i}^{3}-5.874 \cdot d_{i}^{3}+3.726 \cdot l_{i}^{3} \\
& -0.000586 \cdot t_{i} \cdot d_{i} \cdot l_{i} .
\end{aligned}
$$


- Manufacturing cost function for cylindrical feature and position tolerance:

$$
\begin{aligned}
f_{2}\left(t_{i}, d_{i}, l_{i}\right)= & 20.349-88.842 \cdot t_{i}+0.0441 \cdot d_{i}+0.00821 \cdot l_{i} \\
& +135.762 \cdot t_{i}^{2}-0.000218 \cdot d_{i}^{2}+0.000941 \cdot l_{i}^{2} \\
& -0.0237 \cdot t_{i} \cdot d_{i}-0.00449 \cdot t_{i} \cdot l_{i} \\
& -3.0117 \cdot d_{i} \cdot l_{i}-59.925 \cdot t_{i}^{3}+5.354 \cdot d_{i}^{3} \\
& -6.617 \cdot l_{i}^{3}+8.911 \cdot t_{i} \cdot d_{i} \cdot l_{i}
\end{aligned}
$$

where $t_{i}$ is tolerance, $f_{j}(\bullet)$ is the manufacturing cost parameter and tolerance function for model $j$, and $d$ and $l$ are the diameter and length of cylindrical feature.

\subsection{Knowledge Network Modeling for Collaborative Design}

\subsubsection{DESIGN KNOWLEDGE FROM PERFORMANCE}

The overall performance of a complex product generally depends on a number of specifications distributed in various disciplines, such as mechanics, cybernetics, dynamics, and so forth. Design knowledge from performance in various disciplines can be divided into two classes: specification constraints and relation constraints. The former is design goals, including the requirements and limitations of product performance, shape size, and so forth, which are determined by user requirements before starting design process. The latter is relationships between specifications and design variables, which can be obtained from design principles in each discipline. In traditional design, the information in specification constraints are not made for full use, but only treated as evaluated criteria.

\subsubsection{DESIGN KNOWLEDGE FROM SIMULATION}

As the core of knowledge discovery from simulation, an efficient and appropriate data mining (DM) algorithm plays an important role in a successful knowledge discovery in a database (KDD) process. It is very difficult that one object identified by a real value attribute is exactly equal to another in the decision table. So the equivalence relation in basic rough-set theory (RST) is too strict for a quantitative data such as FEA simulation data. In this study, a fuzzy indiscernibility relation is introduced to replace the equivalence relation in basic RST.

For a decision table $S=(U, A \cup\{d\})$, if the value set $V_{a}$ is composed of quantitative value, the value on attribute $a \in A$ can be catalogued into several fuzzy sets described by natural language as 'low', 'normal', or 'high', etc. Assume that the set $L_{a}$ of linguistic terms of attribute $a$ is equal to $\left\{l_{1}^{a}, l_{2}^{a}, \ldots, l_{\left|L_{a}\right|}^{a}\right\}$. Object $x$ belongs to the $l$ th fuzzy set of attribute $a$ with fuzzy function $f_{a l}^{x}$.
For any two objects $x$ and $y$ in $U$, if there exists linguistic term $l$ of attribute $a$ satisfying $f_{a l}^{x}>0$ and $f_{a l}^{y}>0$, it is said that there are fuzzy indiscernibility relations on single attribute $a$ between objects $x$ and $y$. The indiscernibility degree between them on the linguistic term $l$ can be measured by $\mu_{a l}=\min \left(f_{a l}^{x}, f_{a l}^{y}\right)$. Similarly, if the same linguistic terms of an attribute subset $B$ exist in both object $x$ and $y$ with membership values larger than zero, $x$ and $y$ are said to have a fuzzy indiscernibility relation on attribute subset $B$ with the membership value equal to $\mu_{B}=\min \left(\left\{\min \left(f_{a l}^{x}, f_{a l}^{y}\right): l \in L_{a}, a \in B\right\}\right)$

$$
\operatorname{IND}^{\prime}(B)=\left\{\left((x, y), \mu_{B}\right): \forall_{a \in B}\left(f_{a l}^{x}>0, f_{a l}^{y}>0\right) .\right.
$$

According to the above fuzzy similarity relation, the universe $U$ can be partitioned by attribute subset $B$. $[x]_{\mathrm{IND}^{\prime}(B)}$ denotes the fuzzy equivalence class of $\operatorname{IND}^{\prime}(B)$ defined by $x$. Thus fuzzy lower approximation and fuzzy upper approximation of subset $X$ in $U$ are defined as:

$$
\begin{aligned}
& \underline{B}(X)=\left\{\left([x]_{\mathrm{IND}^{\prime}(B)}, \mu_{B}(x)\right): x \in U,[x]_{\mathrm{IND}^{\prime}(B)} \subseteq X\right\} \\
& \bar{B}(X)=\left\{\left([x]_{\mathrm{IND}^{\prime}(B)}, \mu_{B}(x)\right): x \in U,[x]_{\mathrm{IND}^{\prime}(B)} \cap X \neq \Phi\right\} .
\end{aligned}
$$

By computing $\underline{B}\left(C_{k}\right)$ and $\bar{B}\left(C_{k}\right)(1 \leq k \leq r(d))$, certain and possible rules can be induced respectively.

Based on the above theory, the detailed steps of knowledge discovery from the simulation data are summarized as:

Step 1. According to the domain knowledge, decide the center point for fuzzy partition. Adopt a fuzzy member function to transform the quantitative value into several linguistic term descriptions.

Step 2. Compute the decision class $C_{k}$ through decision attribute subset $d$.

Step 3. For any condition attribute subset $B \in \rho(A)$, compute the fuzzy equivalence class $\operatorname{IND}^{\prime}(B)$.

Step 4. For each decision class $C_{k}$, compute $\underline{B}\left(C_{k}\right)$ and $\bar{B}\left(C_{k}\right)$ respectively, and insert them into a certain object set and uncertain object set, respectively.

Step 5. Repeat Steps 3, 4 until all condition attribute subsets and all decision classes have been calculated.

Step 6. The certain rules are induced from certain object sets and the uncertain rules can be induced from an uncertain object set. Calculate each rule's support degree, accuracy, and efficiency measurement.

Step 7. Reduce the rule sets, and then add rules into the fuzzy rule knowledge base. 


\subsection{Knowledge Network Modeling for Concurrent and Collaborative Design}

The knowledge network model includes: (1) Constraints and functions from assembly, manufacture, and performance; and (2) intervals, which are derived from rule sets in the knowledge discovery from simulation. The knowledge network model is used in the following coordination and optimization.

Model I. (The model for coordination). The model for collaboration based on knowledge network is formulated as

$$
\begin{aligned}
& \boldsymbol{x} \in\left[\boldsymbol{x}^{\mathrm{L}}, \boldsymbol{x}^{\mathrm{U}}\right] \\
& \text { s.t. } \begin{cases}\boldsymbol{g}(\boldsymbol{x})=0 & \boldsymbol{g}=\left[g_{1}, g_{2}, \ldots, g_{l}\right]^{\mathrm{T}} \\
\boldsymbol{h}(\boldsymbol{x}) \leq 0 & \boldsymbol{h}=\left[h_{1}, h_{2}, \ldots, h_{m}\right]^{\mathrm{T}} \\
\boldsymbol{x} \in\left[\boldsymbol{x}^{\mathrm{L} 0}, \boldsymbol{x}^{\mathrm{U} 0}\right]\end{cases}
\end{aligned}
$$

where $\boldsymbol{x} \in \boldsymbol{R}^{n}$ is an $n$-dimensional design variable vector; $\boldsymbol{g}$ and $\boldsymbol{h}$ are constraint vectors of equations and inequalities, respectively. $\left[\boldsymbol{x}^{\mathrm{L} 0}, \boldsymbol{x}^{\mathrm{U} 0}\right]$ are initial conditions of the original evaluated intervals of design variables. It is derived from rule sets in the knowledge discovery from simulation, $\left[\boldsymbol{x}^{\mathrm{L}}, \boldsymbol{x}^{\mathrm{U}}\right]$ are consistent intervals filtered by the parameter coordination algorithm which are corresponded to the given design goals. If the domain of any variable in $\boldsymbol{x}$ vector is empty, it means that there exists conflicts in the current design project and some specifications cannot meet the requirements.

After consistent intervals $\left[x^{\mathrm{L}}, \boldsymbol{x}^{\mathrm{U}}\right]$ are filtered by parameter coordination algorithm in the parameter coordination process, an optimization process is implemented based on the knowledge network model and the result of parameter coordination.

Model II. (The model for robust optimization). The model for robust optimization based on knowledge network is formulated as:

Given $\boldsymbol{p}$ and the tolerance of $\boldsymbol{x}$ and $\boldsymbol{p}: \boldsymbol{t}_{x_{1}}, \boldsymbol{t}_{x_{2}}, \boldsymbol{t}_{p_{1}}, \boldsymbol{t}_{p_{2}}$, find $\boldsymbol{x}$

$$
\begin{aligned}
\min \varphi(\boldsymbol{x}, \boldsymbol{p})= & \sum_{i=1}^{q} w_{i}\left[\alpha \frac{\mu_{y_{i}}}{\mu_{i}^{*}}-(1-\alpha) \frac{\sigma_{y_{i}}}{\sigma_{i}^{*}}\right] \\
\text { s.t. } \quad g_{i}(\boldsymbol{x}, \boldsymbol{p})= & 0, \quad i=1, \ldots, l \\
h_{j, \text { new }}(\boldsymbol{x}, \boldsymbol{p})= & h_{j}+\sum_{k=1}^{n}\left|\frac{\partial h_{j}}{\partial x_{k}}\right|_{\mathbf{x}} \mid \cdot \Delta x_{k} \\
& +\sum_{k=1}^{s}\left|\frac{\partial h_{j}}{\partial p_{k}}\right|_{\mathbf{x}} \mid \cdot \Delta p_{k} \leq 0, \quad j=1, \ldots, m \\
& \boldsymbol{x} \in\left[\boldsymbol{x}^{\mathrm{L}}, \boldsymbol{x}^{\mathrm{U}}\right]
\end{aligned}
$$

where $\boldsymbol{x}=\left\{x_{1}, \ldots, x_{n}\right\}$ is a vector of design variables and $\boldsymbol{p}=\left\{p_{1}, \ldots, p_{s}\right\}$ is a vector of design parameters whose values are fixed as a part of the problem specifications. $\boldsymbol{t}_{x_{1}}, \boldsymbol{t}_{x_{2}}, \boldsymbol{t}_{p_{1}}, \boldsymbol{t}_{p_{2}}$ are tolerance of design variables and design parameters, respectively. $\boldsymbol{y}=\boldsymbol{y}(\boldsymbol{x}, \boldsymbol{p})$ is a vector of the system performance. $\mu_{y}, \sigma_{y}$ are the mean value and the standard deviation of $y$, respectively. $\mu^{*}, \sigma^{*}$ are the expected mean value and the expected standard deviation. $\alpha$ is the weight factor. $g_{i}(\boldsymbol{x}, \boldsymbol{p})$ and $h_{j, \text { new }}(\boldsymbol{x}, \boldsymbol{p})$ are constraints in the robust optimization model, where $\Delta \boldsymbol{x}=\boldsymbol{t}_{x_{1}}-\boldsymbol{t}_{x_{2}}$, $\Delta \boldsymbol{p}=\boldsymbol{t}_{p_{1}}-\boldsymbol{t}_{p_{2}}$

\section{Parameter Coordination}

The algorithm utilized to solve the knowledge network model can filter out the redundant region through the initial domain of design variables and obtain the consistency solution space corresponding to the given knowledge.

A temporary vector $\boldsymbol{\theta}$ is constructed for inequality constraint vector $\boldsymbol{h}, \boldsymbol{\theta} \in R^{n}$ and $\boldsymbol{\theta} \geq 0$. With the help of $\boldsymbol{\theta}$, inequalities can be transformed into equalities:

$$
\boldsymbol{h}(\boldsymbol{x}) \leq 0 \Rightarrow \boldsymbol{h}(\boldsymbol{x})+\boldsymbol{\theta}=0
$$

The relationship matrix for the coordination model is defined as:

$$
\boldsymbol{F}=\{f(i j)\} \quad f(i j)= \begin{cases}1, & f_{i} \text { contains } x_{j} \\ 0, & f_{i} \text { does not contains } x_{j}\end{cases}
$$

where $1 \leq i \leq l+m, 1 \leq j \leq n+m$.

In addition, two secondary vectors, $\boldsymbol{c}_{r}$ and $\boldsymbol{d}_{s}$, are defined as:

$$
\begin{aligned}
\boldsymbol{c}_{r} & =\left[c_{r}(1), c_{r}(2), \ldots, c_{r}(n+m)\right] \\
c_{r}(\lambda) & =\left\{\begin{array}{lll}
1, & \lambda=r \\
0, & \lambda \neq r
\end{array} \quad r=1,2, \ldots, n+m .\right. \\
\boldsymbol{d}_{s} & =\left[d_{s}(1), d_{s}(2), \ldots, d_{s}(l+m)\right] \\
d_{s}(\lambda) & =\left\{\begin{array}{ll}
1, & \lambda=s \\
0, & \lambda \neq s
\end{array} \quad s=1,2, \ldots, l+m .\right.
\end{aligned}
$$

The parameter coordination algorithm used to solve the parameter coordination model is described as:

Algorithm $A$ :

Procedure LabelResolve $\left(\operatorname{In}\left\{\mathrm{C}=\left[f_{i}^{j}\right], x=0, y=\left[x^{L 0}\right.\right.\right.$, $\left.\left.\left.x^{U 0}\right]\right\} ; \operatorname{Out}\{y\}\right)$ 


\author{
BEGIN \\ WHILE $(y \neq x)$ DO \\ $x=y$ \\ FOR $j=1$ TO $n+m$ \\ FOR $i=1$ TO $l+m$ \\ IF $\left(c_{i} F d_{j} \neq 0\right)$ \\ $y_{i}=f_{i}^{j}(x)$ \\ ENDIF \\ IF $y_{j}==\{$ null set $\}$ \\ Exit \\ Show conflict message \\ ENDIF \\ END FOR \\ END FOR \\ ENDWHILE \\ END \\ END Procedure
}

\section{Robust Parameter Optimization}

The penalty function method is adopted to modify the robust optimization model (Equation (13)), and the objective function $f$ is formulated as:

$f(\boldsymbol{x}, \boldsymbol{p})=\varphi(\boldsymbol{x}, \boldsymbol{p})-\sum_{i=1}^{l} K_{i} \Phi\left(g_{i}(\boldsymbol{x}, \boldsymbol{p})\right)-\sum_{j=l+1}^{l+m} K_{j} \Theta\left(h_{j, \text { new }}(\boldsymbol{x}, \boldsymbol{p})\right)$

where $K_{i}$ and $K_{j}$ are penalty factors.

Robustness for the system performance function is formulated as:

$$
\left\{\begin{array}{c}
\varphi(\boldsymbol{x}, \boldsymbol{p})=\sum_{i=1}^{q} w_{i}\left[\alpha \frac{\mu_{y_{i}}}{\mu_{i}^{*}}+(1-\alpha) \frac{\sigma_{y_{i}}}{\sigma_{i}^{*}}\right] \\
y_{i}=y_{i}(\boldsymbol{x}, \boldsymbol{p}), i=1, \ldots, q .
\end{array}\right.
$$

Equation (19) is solved by Monte Carlo method. Let design solution point $A$ be $\boldsymbol{x}=\boldsymbol{x}_{0}, \boldsymbol{p}=\boldsymbol{p}_{0}$, and tolerance of $\boldsymbol{x}, \boldsymbol{p}$ be $\Delta \boldsymbol{x}$ and $\Delta \boldsymbol{p}$. First, the stochastic points $\boldsymbol{x}_{i}$ and $\boldsymbol{p}_{i}(i=1, \ldots, k)$ are sampled around $\boldsymbol{x} \in[\boldsymbol{x}-\Delta \boldsymbol{x}, \boldsymbol{x}+\Delta \boldsymbol{x}]$ and $\boldsymbol{p} \in[\boldsymbol{p}-\Delta \boldsymbol{p}, \boldsymbol{p}+\Delta \boldsymbol{p}]$. Then, calculate $y$ for all the stochastic points. Finally, calculate the mean value $\mu_{y}$ and the standard deviation $\sigma_{y}$ of $y$ in the $\boldsymbol{x} \in[\boldsymbol{x}-\Delta \boldsymbol{x}, \boldsymbol{x}+\Delta \boldsymbol{x}]$ and $\boldsymbol{p} \in[\boldsymbol{p}-\Delta \boldsymbol{p}, \boldsymbol{p}+\Delta \boldsymbol{p}]$.

For input $\boldsymbol{x}=\boldsymbol{x}_{0}, \boldsymbol{p}=\boldsymbol{p}_{0}, \Delta \boldsymbol{x}$ and $\Delta \boldsymbol{p}, g_{i}$ and $h_{j, \text { new }}$ in the model (Equation (18)) are calculated. Then, penalty function $\Theta\left(h_{j, \text { new }}(\boldsymbol{x}, \boldsymbol{p})\right)$ and $\Phi\left(g_{i}(\boldsymbol{x}, \boldsymbol{p})\right)$ are redefined as:

$$
\begin{aligned}
\theta\left(h_{j, \text { new }}(\boldsymbol{x}, \boldsymbol{p})\right) & =\left\{\begin{array}{cc}
h_{j, \text { new }}(\boldsymbol{x}, \boldsymbol{p}) & \text { if } h_{j, \text { new }}(\boldsymbol{x}, \boldsymbol{p})>0 \\
0 & \text { if } h_{j, \text { new }}(\boldsymbol{x}, \boldsymbol{p}) \leq 0
\end{array}\right. \\
\Phi\left(g_{i}(\boldsymbol{x}, \boldsymbol{p})\right) & =\left|g_{i}(\boldsymbol{x}, \boldsymbol{p})\right| .
\end{aligned}
$$

Based on the above robustness analysis, the robust optimization model can be solved by genetic algorithm. Variables and constraints have been defined in the parameter coordination model (Equation (12)). Search space is the consistent intervals $\left[\boldsymbol{x}^{\mathrm{L}}, \boldsymbol{x}^{\mathrm{U}}\right]$, which are filtered by the parameter coordination algorithm in the parameter coordination process. So the parameter coordination to obtain the consistent solution space in Section 3 is the basis of robust design optimization in this section. According to Equation (13), the parameter coordination model (Equation (12)) is transferred to the robust optimization model. The penalty function is calculated by Equations (20) and (21). The objective function is calculated by Equation (18), which is expressed as fitness. Selection, crossover, and mutation are implemented to obtain the optimization solutions.

\section{Knowledge Network Driven Concurrent and Collaborative Design System Architecture}

An Internet based system architecture is proposed in this study to support concurrent and collaborative parameter design. The architecture is structured in a three-layered framework: information, application, and end user layer. In such a system, the end user layer is situated in the user's desktop and is connected to the application Web server (application layer), which in turn is connected to the information databases (information layer).

\subsection{Information layer}

Coordination and optimization during parameter design is difficult as decisions need to be taken in collaboration with other teams that do not have access to the knowledge of their distributed partners. To overcome this issue, it is necessary to have a distributed source of knowledge to support the different activities. The knowledge network model from assembly, manufacturing, performance, and simulation addresses such requirement because it is an information model that captures concurrent and collaborative processes. Its knowledge data integrity is captured as a result of the way the model represents the constraints, functions, and intervals.

\subsection{Application layer}

The application layer consists of two elements: (1) parameter coordination and robust optimization applications provides a range of key product life cycle applications that need to be preformed in a collaborative manner. This research is concerned with the parameter design; hence the proposed applications include parameter coordination and robust 
optimization and (2) knowledge management applications control knowledge access, maintain the knowledge, and manage the geographically distributed collaborative team.

\subsection{End user layer}

The end user layer forms the front end of the system. It consists mainly of a web browser, such as Internet Explorer or Netscape, to view and use the different decision support engineering applications and collaborative tools.

\section{Design Example}

The following is an example of a piston-connecting mechanism of an automotive engine shown in Figure 4. This example is to illustrate how to design the parameter and tolerance with three performance targets: assembly functional requirements, manufacturing cost requirements, and weight requirement. Designed parameters are the outside diameter of piston $x_{1}$, length of piston $x_{2}$, diameter of piston pin hole $x_{3}$, diameter of piston pin $x_{4}$, length of piston pin $x_{5}$, diameter of connecting rod small end bearing $x_{6}$, diameter of connecting rod big end bearing $x_{7}$, width of connecting rod $x_{8}$, and center distance of connecting rod small end bearing and big end bearing $x_{9}$. Designed tolerances are tolerance of outside diameter of piston $t_{1}$, tolerance of diameter of piston pin hole $t_{2}$, tolerance of diameter of piston pin $t_{3}$, tolerance of diameter of connecting rod small end bearing $t_{4}$, tolerance of diameter of connecting rod big end bearing $t_{5}$, and tolerance of center distance of connecting rod small end bearing and big end bearing $t_{6}$.

\subsection{Knowledge Network Modeling}

Design knowledge from assembly.

Design knowledge from assembly includes parameter constraints $g$, variation functions $f$, and tolerance constraints $\boldsymbol{h}$ as follows.

$$
\left\{\begin{array}{l}
g_{1}\left(x_{3}, x_{4}\right)=x_{4}-x_{3}=0.01 \\
g_{2}\left(x_{4}, x_{6}\right)=x_{6}-x_{4}=0.03
\end{array}\right.
$$
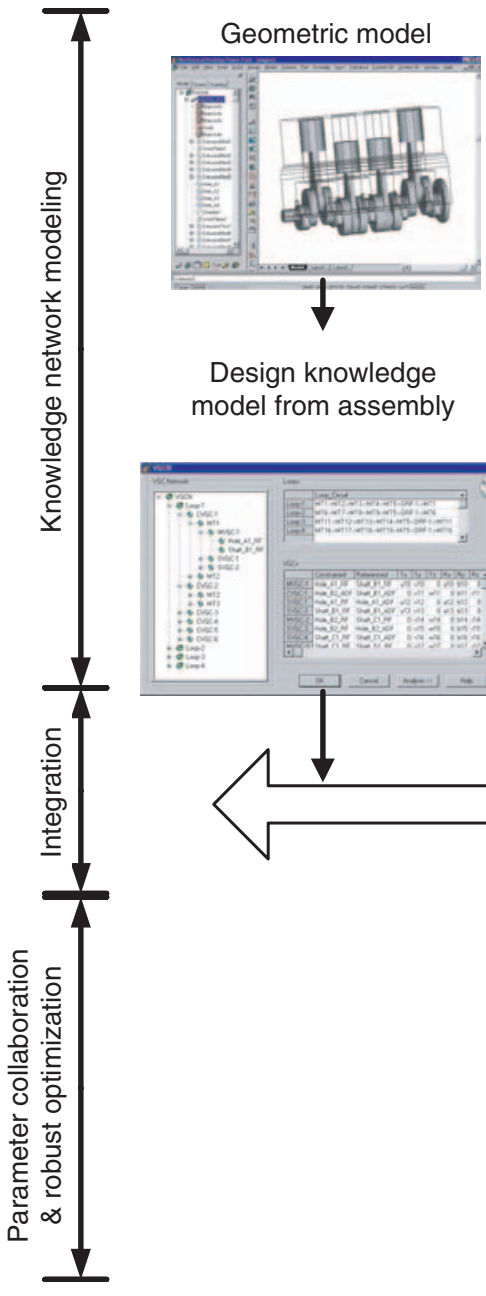
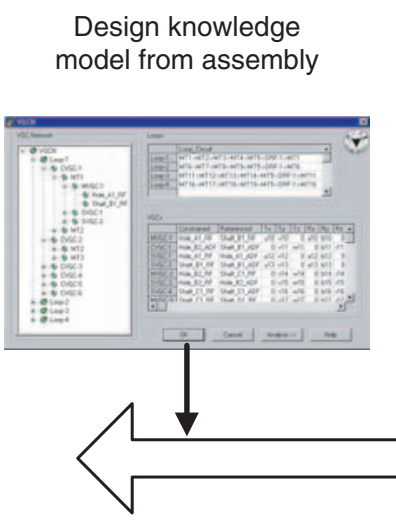

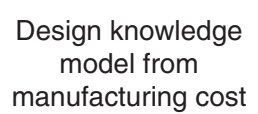

manufacturing cost

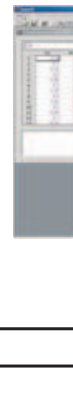

\section{Design knowledge model from performance}

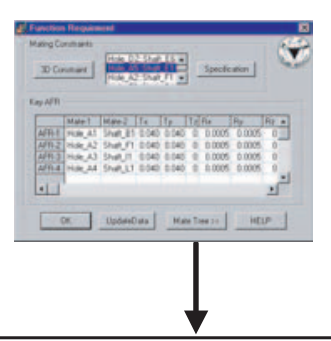

FEA model

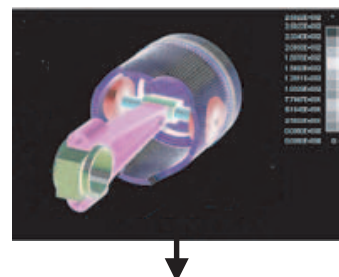

Design knowledge model from simulation

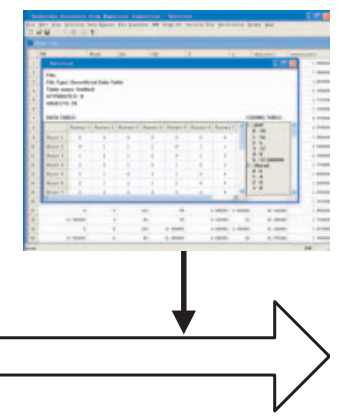

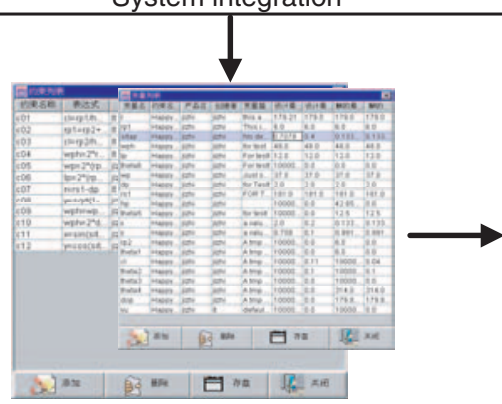

Parameter coordination

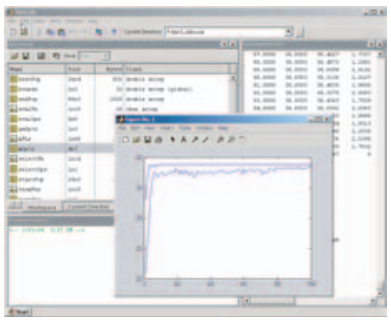

Robust optimization

Figure 4. A design example of piston-connecting mechanism of automotive engine. 


$$
\begin{aligned}
& \left\{\begin{array}{l}
f_{S 1}\left(v_{2}, v_{3}, \gamma_{2}, \gamma_{3}, x_{5}, x_{8}\right) \\
=\left\{\left[v_{2}+\left(x_{5}-x_{8}\right) \cdot \gamma_{2}\right]^{2}+\left[v_{3}+\left(x_{5}-x_{8}\right) \cdot \gamma_{3}\right]^{2}\right\}^{1 / 2} \\
f_{S 2}\left(w_{2}, w_{3}, \beta_{2}, \beta_{3}, x_{5}, x_{8}\right) \\
=\left\{\left[w_{2}-\left(x_{5}-x_{8}\right) \cdot \beta_{2}\right]^{2}+\left[w_{3}-\left(x_{5}-x_{8}\right) \cdot \beta_{3}\right]^{2}\right\}^{1 / 2} \\
\ldots \ldots
\end{array}\right. \\
& \left\{\begin{array}{c}
h_{1}\left(u_{1}, v_{1}, \alpha_{1}, \beta_{1}, x_{2}, t_{1}\right) \\
=\left(u_{1}+x_{2} \cdot \beta_{1}\right)^{2}+\left(v_{1}+x_{2} \cdot \alpha_{1}\right)^{2}-\left(t_{1} / 2\right)^{2} \leq 0 \\
h_{2}\left(v_{2}, w_{2}, \beta_{2}, \gamma_{2}, x_{5}, x_{8}, t_{2}\right) \\
=\left[v_{2}+\left(x_{5}-x_{8}\right) \cdot \gamma_{2}\right]^{2} \\
\quad+\left[w_{2}+\left(x_{5}-x_{8}\right) \cdot \beta_{2}\right]^{2}-\left(t_{2} / 2\right)^{2} \leq 0 \\
\ldots \cdots \\
h_{6}\left(v_{6}, w_{6}, t_{6}\right)=\left(v_{6}\right)^{2}+\left(w_{6}\right)^{2}-\left(t_{6} / 2\right)^{2} \leq 0 .
\end{array}\right.
\end{aligned}
$$

Design knowledge from manufacture.

Regression analysis is used to summarize a collection of sampled data by fitting it to a model that will accurately describe the data. Each regression model has adjustable coefficients, which can be adjusted in order to achieve close agreement between values of the regression model and the sampled data. Regression analysis can turn the sampled data points into a smooth continuous function. Manufacturing cost functions in this example are as follows:

$$
\left\{\begin{array}{c}
f_{C 1}\left(t_{1}, x_{1}, x_{2}\right)=84.708-277.022 \cdot t_{1}+0.0938 \cdot x_{1} \\
\quad-0.00396 \cdot x_{2}+163.566 \cdot t_{1}^{2}+0.00135 \cdot x_{1}^{2} \\
\quad+0.000507 \cdot x_{2}^{2}+0.0262 \cdot t_{1} \cdot x_{1}+0.0208 \cdot t_{1} \cdot x_{2} \\
\quad+0.000245 \cdot x_{1} \cdot x_{2}+110.645 \cdot t_{1}^{3}-5.874 \cdot x_{1}^{3} \\
\quad+3.726 \cdot x_{2}^{3}-0.000586 \cdot t_{1} \cdot x_{1} \cdot x_{2} \\
f_{C 2}\left(t_{2}, x_{3}, x_{5}, x_{8}\right)=87.241-277.813 \cdot t_{2}+0.0167 \cdot x_{3} \\
\quad+0.0449 \cdot\left(x_{5}-x_{8}\right)+166.482 \cdot t_{2}^{2}+0.00738 \cdot x_{3}^{2} \\
\quad-0.000845 \cdot\left(x_{5}-x_{8}\right)^{2}+0.0498 \cdot t_{2} \cdot x_{3} \\
\quad+0.0266 \cdot t_{2} \cdot\left(x_{5}-x_{8}\right)+0.000242 \cdot x_{3} \cdot\left(x_{5}-x_{8}\right) \\
\quad+106.362 \cdot t_{2}^{3}-8.238 \cdot x_{3}^{3}+1.417 \cdot\left(x_{5}-x_{8}\right)^{3} \\
\quad-0.00108 \cdot t_{2} \cdot x_{3} \cdot\left(x_{5}-x_{8}\right) \\
\quad \ldots \ldots
\end{array}\right.
$$

Design knowledge from performance.

Using the above regression analysis, we obtain design knowledge from performance in this example, which is a function for weight as follows:

$$
\begin{aligned}
f_{G}( & \left.x_{1}, x_{2}, x_{3}, x_{4}, x_{5}, x_{6}, x_{7}, x_{8}, x_{9}\right) \\
= & c_{0}+c_{1} \cdot x_{1}+c_{2} \cdot x_{2}+c_{3} \cdot x_{3}+c_{4} \cdot x_{4} \\
& +c_{5} \cdot x_{5}+c_{6} \cdot x_{6}+c_{7} \cdot x_{7}+c_{8} \cdot x_{8}+c_{9} \cdot x_{9}
\end{aligned}
$$

where $c_{0}, \ldots, c_{9}$ are coefficients.

Design knowledge from simulation.

In this study, the commercial finite-element program ANSYS is used for the analysis of the piston-connecting mechanism. The simulation is carried out with different parameters. Then the concerned FEA data are collected into a decision table. According to the domain knowledge, each of the parameters is discretized into three levels. A Boolean Reasoning discretization method is carried out to discretize other condition attributes into several levels. The knowledge discovery method, which is shown in Section 2.2.2, is adopted to acquire explicit rules. It can deal with continuous data in simulation results and does not depend much on prior knowledge. Based on the acquired rules, the initial intervals $\left[x^{\mathrm{L} 0}, x^{\mathrm{U} 0}\right]$ derived from rule sets in this example are as follows:

$$
\begin{aligned}
& x_{1} \in[80,91], x_{2} \in[88,97], x_{3} \in[16,27], x_{4} \in[16,27], \\
& x_{5} \in[82,92], x_{6} \in[14,26], \\
& x_{7} \in[42,58], x_{8} \in[31,45], x_{9} \in[141,166], \\
& t_{1} \in[0.002,0.050], t_{2} \in[0.002,0.012], \\
& t_{3} \in[0.001,0.012], t_{4} \in[0.005,0.080], t_{5} \in[0.003,0.050], \\
& t_{6} \in[0.003,0.600] .
\end{aligned}
$$

\subsection{Parameter Coordination}

Based on the above knowledge network, Model I for collaboration is constituted by Equation (12). The parameter coordination algorithm is utilized to solve Model I to obtain the consistency intervals as follows:

$$
\begin{aligned}
& x_{1} \in[84,87], x_{2} \in[92,96], x_{3} \in[18,21], x_{4} \in[18,21], \\
& x_{5} \in[83,86], x_{6} \in[18,21], x_{7} \in[48,52], x_{8} \in[36,40], \\
& x_{9} \in[150,154], t_{1} \in[0.005,0.035], \\
& t_{2} \in[0.004,0.009], t_{3} \in[0.002,0.007], t_{4} \in[0.010,0.030], \\
& t_{5} \in[0.005,0.030], t_{6} \in[0.010,0.300] .
\end{aligned}
$$

\subsection{Robust Optimization}

Based on Model I and consistency intervals, Model II for robust optimization is established by Equation (13). The genetic arithmetic is used to optimize Model II. The final optimized parameters and tolerances are shown as:

$$
\begin{aligned}
& x_{1}=85.9, x_{2}=95.3, x_{3}=19.99, x_{4}=20.0, x_{5}=84.2, \\
& x_{6}=20.03, x_{7}=50.9, x_{8}=39.0, x_{9}=152.0, t_{1}=0.02, \\
& t_{2}=0.007, t_{3}=0.005, t_{4}=0.017, t_{5}=0.015, t_{6}=0.1 .
\end{aligned}
$$




\subsection{Discussion on the Example}

Sections 6.1-6.3 demonstrate a case study to corroborate the efficacy of the proposed method. The knowledge network reduces the possibility of iterative design. The input of Model I for collaboration is design knowledge from assembly, manufacture, performance, and simulation. The output is the filtered design intervals, in which conflict parameters have been removed. This design example is a typical concurrent and collaborative design problem with uncertainties. The robust optimization method based on Model II achieves a robust solution for the parameter uncertainties. The method described in this study was used to develop a knowledge network driven concurrent and collaborative design system, which is now running in an automotive engine design project.

\section{Conclusions}

This study has shown how to design parameters of a complex product using knowledge network modeling, coordination, and a robust optimization approach. Knowledge network includes design knowledge from assembly, manufacture, performance, and simulation. As opposed to the traditional concurrent modeling methodology, the proposed knowledge network method can be used to model 'time dimension' concurrence and 'space dimension' collaboration in the development process of a complex product. Based on Model I for collaboration, a parameter coordination algorithm was designed using interval arithmetic to refine the intervals. The algorithm can be used to verify the design process early in the process and to assist the designers in determining design variables to reduce the multidisciplinary iterations in concurrent and collaborative design. Based on Model II for robust optimization, a robust optimization algorithm was presented to obtain an optimized product solution. The proposed method has addressed the parameter coordination and robust optimization simultaneously. Further research will focus on system integration, which is shown in Figure 4, including information and knowledge integration.

\section{Acknowledgments}

This research is supported by the National Natural Science Foundation of China (Grant No. 50575142 and 60304015) and the Shanghai Committee of Science and Technology (No. 055107048 and 04ZR14081).

\section{References}

1. Fonseca, C.M. and Fleming, P.J. (1995). An Overview of Evolutionary Algorithms in Multiobjective Optimization, Evolutionary Computation, 3(1): 1-16.

2. Coello, C.A. (1999). A Comprehensive Survey of Evolutionary Based Multiobjective Optimization Techniques, Knowledge and Information Systems An International Journal, 1(3): 269-308.

3. Fonseca, C.M. and Fleming, P.J. (1993). Genetic Algorithms for Multiobjective Optimization: Formulation, Discussion and Generalization, In: Stephanie, F. (ed.), Proceedings of the Fifth International Conference on Genetic Algorithms, pp. 416-423, San Mateo, California.

4. Narayanan, S. and Azarm, S. (1999). On Improving Multiobjective Genetic Algorithms for Design Optimization, Structural Optimization, 18(2-3): 146-155.

5. Kurapati, A., Azarm, S. and Wu, J. (2000). Constraint Handling in Multiobjective Genetic Algorithms, In: Proceedings of 8th AIAA/NASA/USAF/ISSMO Symposium on Multidisciplinary Analysis and Optimization, Long Beach, CA, Paper No. AIAA-20004893.

6. Le, T.V. (1995). A Fuzzy Evolutionary Approach to Constrained Optimization Problems, In: Proceedings of the Second IEEE Conference on Evolutionary Computation, pp. 274-278, Perth, Australia.

7. Hajela, P. and Lee, J. (1996). Constrained Genetic Search via Schema Adaptation. An Immune Network Solution, Structural Optimization, 12(1): 11-15.

8. Coello, C.A. (1999). A Survey of Constraint Handling Techniques used with Evolutionary Algorithms, Technical Report Lania-RI-99-04, Laboratorio Nacional De Informatica Avanzada, Veracruz, Mexico.

9. Michalewicz, Z. (1995). A Survey of Constraint Handling Techniques in Evolutionary Computation Methods, In: Proceedings of the Fourth Annual Conference on Evolutionary Programming, pp. 135-155, MIT, Cambridge, MA.

10. Bloebaum, C.L., Hajela, P. and Sobieski, J. (1992). Nonhierarchic System Decomposition in Structural Optimization, Eng. Optim., 19(4): 171-186.

11. Renaud, J.E. and Tappeta, R.V. (1997). Multiobjective Collaborative Optimization, Trans. ASME, J. Mech. Des., 119(3): 403-411.

12. Kroo, I., Altus, S., Braun, R., Gage, P. and Sobieski, I. (1994). Multidisciplinary Optimization Methods for Aircraft Preliminary Design, In: Proceedings of 5th AIAA/USAF/NASA/ISSMO Symp. MAO, pp. 697-707, Panama City, FL.

13. Braun, R.D., Kroo, I.M. and Moore, A.A. (1996). Use of the Collaborative Optimization Architecture for Launch Vehicle Design, In: Proceedings of 6th AIAA/ USAF/NASA/ISSMO Symp. MAO, pp. 306-318, Bellevue, WA.

14. Kroo, I.M. (1997). Multidisciplinary Optimization Applications in Preliminary Design - Status and Directions, In: Proceedings of 38th AIAA/ASME/ASCE/ AHS /ASC, Structural Dyn. Materials Conf., pp. 414-421, Kissimmee, FL.

15. Serrano, D. and Gossard, D. (1992). Tools and Techniques for Conceptual Design, In: Tong, C. and Sriram, D. (eds), 
Artificial Intelligence in Engineering Design, pp. 71-116, Academic, New York.

16. Kannapan, S.M. and Marshek, K.M. (1993). An Approach to Parametric Machine Design and Negotiation in Concurrent Engineering, In: Kusiak, A. (ed.), Concurrent Engineering: Automation, Tools and Techniques, pp. 509-533, New York: Wiley.

17. Kusiak, A. and Wang, J. (1995). Dependency Analysis in Constraint Negotiation, IEEE Trans. Syst., Man, Cybern., 25(9): 1301-1313.

18. Chen, W. and Lewis, K. (1999). A Robust Design Approach for Achieving Flexibility in Multidisciplinary Design, AIAA Journal, 37(8): 982-990.

19. Lottaz, C. and Robert, Y. (2000). Constraintbased Support for Negotiation in Collaborative Design, Artificial Intelligence in Engineering, 14(3): 261-280.

20. Chang, T.S., Ward, A.C. and Lee, J. (1994). Distributed Design with Conceptual Robustness: A Procedure based on Taguchi's Parameter Design, In: Gadh, R. (ed.), ASME Concurrent Product Design Conf., Vol. 74, pp. 19-29, ASME, Chicago, IL.

\section{Jie Hu}

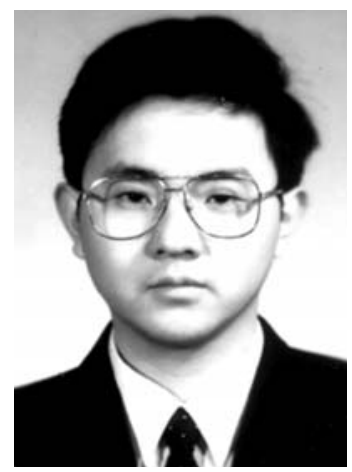

Jie $\mathrm{Hu}$ received his $\mathrm{PhD}$ from Zhejiang University, Hangzhou, China, in 2001. $\mathrm{He}$ worked at Tsinghua University for postdoctoral research from 2001 to 2003. $\mathrm{He}$ is currently an associate professor at Shanghai Jiaotong University. He is leading the National Natural Science Foundation of China under Grant No. 60304015 and 50575142. He has developed methods for areas, such as integration modeling, collaboration, optimization, and simulation of the product development process. He is interested in concurrent and collaborative design and manufacturing. He has published approximately 40 papers in journals and conferences.

\section{Yinghong Peng}

Yinghong Peng received his $\mathrm{PhD}$ from Central South

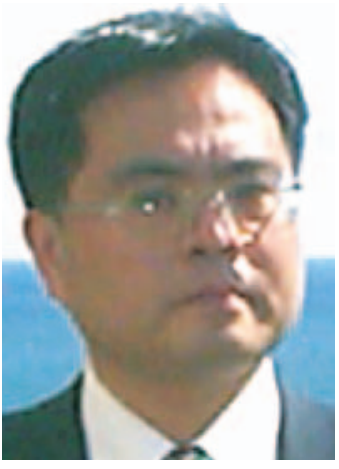
University, Changsha, China, in 1993. He worked at Shanghai Jiaotong University for postdoctoral research from 1993 to 1995 . He is currently a professor at Shanghai Jiaotong University. He has been director at the Institute of Knowledge-Based Engineering of Shanghai Jiaotong University since 2002. He has authorized and edited two books and has published approximately 80 papers in journals and conferences.

\section{Guangleng Xiong}

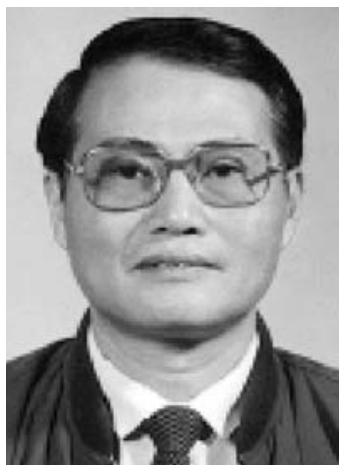

Guangleng Xiong received his BS from Tsinghua University, Beijing, China, in 1959. He is currently a professor at Tsinghua University. He has been chief Engineer at the National CIMS Engineering and Research Center, Beijing, China, since 1987. He also successfully led the planning and implementation of the CIMS project at Chengdu Airplane Factory. $\mathrm{He}$ has authorized and edited eight books and has published approximately 70 papers in journals and conferences. 